Research article

\title{
Optical wavelength dependence of photoacoustic signal of gold nanofluid
}

\author{
Marco Gandolfi ${ }^{\mathrm{a}, \mathrm{b}, \mathrm{c}, \mathrm{d}, \mathrm{e}, *,}$, Francesco Banfi ${ }^{\mathrm{e}, \mathrm{f}}$, Christ Glorieux ${ }^{\mathrm{c}}$ \\ ${ }^{\text {a }}$ CNR-INO, Via Branze 45, 25123 Brescia, Italy \\ ${ }^{\mathrm{b}}$ Department of Information Engineering, University of Brescia, Via Branze 38, 25123 Brescia, Italy \\ ${ }^{\mathrm{c}}$ Laboratory of Soft Matter and Biophysics, Department of Physics and Astronomy, KU Leuven, Celestijnenlaan 200D, B-3001 Leuven, Belgium \\ ${ }^{\mathrm{d}}$ Dipartimento di Matematica e Fisica, Università Cattolica del Sacro Cuore, Via Musei 41, 25121 Brescia, Italy \\ ${ }^{\mathrm{e}}$ Interdisciplinary Laboratories for Advanced Materials Physics (I-LAMP), Via Musei 41, 25121 Brescia, Italy \\ ${ }^{\mathrm{f}}$ FemtoNanoOptics group, Université de Lyon, CNRS, Université Claude Bernard Lyon 1, Institut Lumière Matière, F-69622 Villeurbanne, France
}

\section{A R T I C L E I N F O}

\section{Keywords:}

Photoacoustic effect

Heat transfer

Absorption coefficient

Non-linearity

Kapitza resistance

Nanoparticles

Surface plasmon resonance

\begin{abstract}
A B S T R A C T
We investigate the optical wavelength dependence of the photoacoustic (PA) signal, detected with bandwidth (BW) in the $\mathrm{MHz}$ range, of gold nanospheres (NSs) immersed in water upon illumination with ns laser pulses. We compare the wavelength dependence of the PA signal (within the $\mathrm{MHz} \mathrm{BW}$ ) with the one of the optical absorption coefficient as determined from optical transmission measurements. Thermal boundary conductance (TBC) at the gold-water interface is taken into account, as well as the temperature dependence of the thermal expansion coefficient of water. The effects of NS size and laser pulse duration on the PA signal are also explored. The PA signal is investigated with an opto-thermo-acoustic model considering light absorption in gold NS and in a surrounding water shell.
\end{abstract}

\section{Introduction}

The photoacoustic (PA) effect is based on the generation of acoustic waves upon absorption of light $[1,2]$. In a nutshell, the mechanism is based on the conversion of the absorbed light into heat, yielding a temperature rise inside the sample. This results in acoustic waves [3,4] generated by the thermal expansion of the heated medium (a sketch of the effect is reported in Fig. 1a). Imaging techniques based on the PA effect are intrinsically non destructive and have shown particularly suitable for imaging biological tissues [5,6], the detection of the generated pressure waves unveiling thermophysical, optical and elastic properties of the investigated medium.

Previous works demonstrated that adding exogenous agents in the biological tissue can enhance PA contrast [7-11]. In particular, noble metal nanoparticles have been used as exogenous agents to enhance the PA contrast in imaging applications [12-15]. Metal nanoparticles optical absorption is strongly enhanced at the localised surface plasmon resonance (SPR) $[16,17]$, possibly resulting in the generation of high amplitude pressure waves upon illumination with moderate light fluences. This fact yields a well detectable PA signal, a key feature for PA imaging. In order to further enhance the efficiency of the PA effect generation, several shapes for the plasmonic nanoparticles (NPs) have been proposed, ranging from gold nanospheres (NSs) to nano-rods, bare or coated with $\mathrm{SiO}_{2}$ [18-25].

The information content, obtained by PA imaging, can be enhanced combining images obtained at different optical excitation wavelengths. For instance, multi-spectral optoacoustic tomography (MSOT) has been exploited to disclose information on the composition of biological tissues. This was made possible exploiting the specific absorption spectra of both endogenous and exogenous chromophores [26-28]. PA imaging of the spatial distribution of functionalized NPs has been successfully applied for tumor localization [29]. MSOT has also been shown to be effective in performing functional imaging. MSOT, discriminating between oxygenated and deoxygenated hemoglobin, highlights regions of intense aerobic metabolic activity. Attempts have been made to exploit the subtle temperature dependence of the optical absorption spectrum of nanoparticles for extracting spatially resolved temperature information from PA signal amplitude maps. In this context, an unexpected lack of proportionality between the PA signal and the sample's absorption coefficient, has been reported [30]. In order to make further progress, it is necessary to unveil the relation between the exciting optical wavelength and the PA signal generated by water-immersed NPs.

For typical light pulse durations in the order of a few ns, the

\footnotetext{
* Corresponding author at: CNR-INO, Via Branze 45, 25123 Brescia, Italy.

E-mail address: marco.gandolfi@ino.cnr.it (M. Gandolfi).
} 

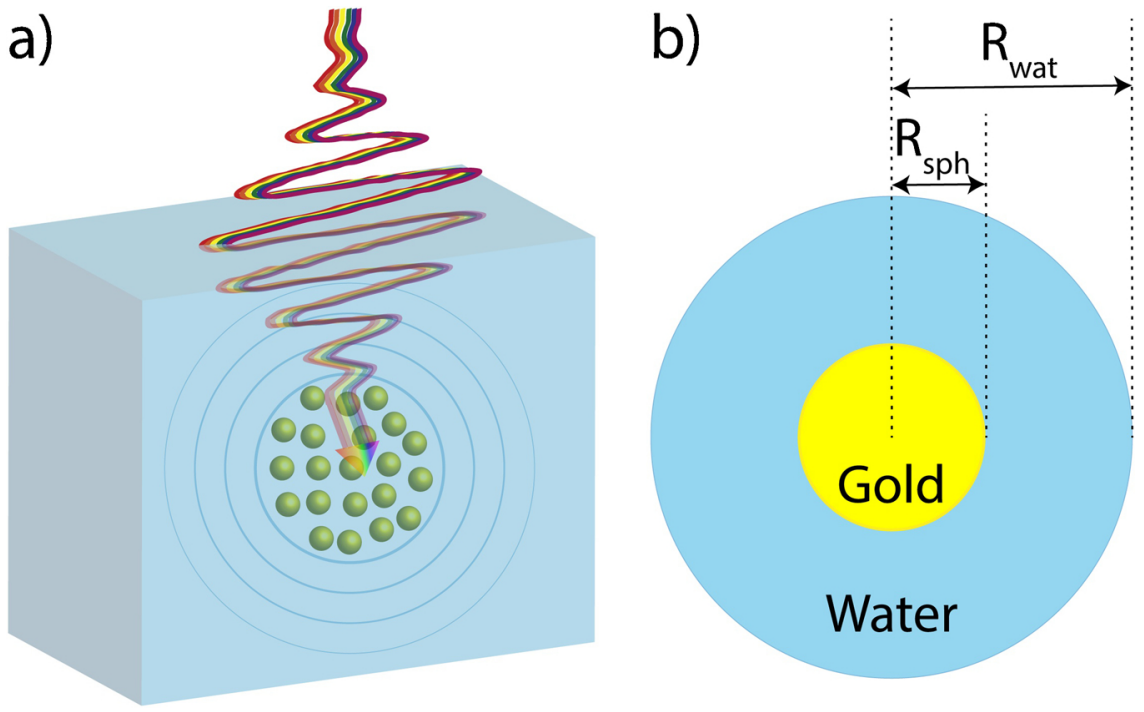

Fig. 1. (a) Schematics of the PA effect. An ensemble of gold NSs immersed in water is illuminated by a short laser pulse. The light absorption causes a temperature increase in both media, generating thermal strains both in the gold NSs and in the surrounding water. Thermal expansion launches pressure waves in the liquid, which compose the PA signal. (b) Cross-section of the geometry used for numerical computation: a gold NS of radius $R_{s p h}$ is surrounded by a spherical shell of water, whose external radius is $R_{\text {wat }}$. For the sake of visualization, the two panels are not to scale. In the current manuscript, the dimension of the gold NS ranges between 5 and $100 \mathrm{~nm}$, while the average interparticle distance is $2 R_{\text {wat }} \sim 5 \mu \mathrm{m}$. hypotheses of thermal and stress confinement [5] do not hold for the case of biological tissues containing dispersed NPs [31]. Thus, the commonly used expression, linearily linking the pressure rise $p$ to the optical fluence $\Phi\left(\mathrm{J} / \mathrm{m}^{2}\right)$ [5], $p=\Gamma \beta \Phi$, with $\Gamma$ the dimensionless Grüneisen parameter ${ }^{1}$ and $\beta$ the absorption coefficient $\left(\mathrm{m}^{-1}\right)$, may not be used. A more comprehensive modelling is thus required to describe the PA effect.

The PA effect involves acoustic waves launched by thermal expansion of both the NPs and the surrounding water upon light illumination. Modelling these processes requires accounting for the interplay among optics (to quantify light absorption), thermics (to access the temperature increase) and acoustics (to finally calculate the pressure wave).

Light absorption can lead to a high local temperature increase both in the NP [32] and in the surrounding liquid. The temperature can range up to few hundreds $\mathrm{K}$ [33], even upon moderate light fluence. Such high temperatures may well imply a strong variation of the thermal and mechanical properties.

Among all the temperature dependent physical parameters, the water thermal expansion coefficient $\alpha_{\text {wat }}$ displays the strongest temperature dependence. In regimes where the temperature dependence of $\alpha_{\text {wat }}$ has to be considered, the pressure wave equation becomes nonlinear with respect to temperature $[34,35]$. Hence, the PA signal becomes non-linear with light fluence $[31,36]$. Another aspect, which needs to be accounted for, is the thermal boundary conductance (TBC), also addressed as Kapitza's conductance, ruling the heat transfer at the interface between the NPs and the liquid. The estimation of TBC is still a debated issue, since the latter strongly depends on the involved materials combination $[37,38]$, on temperature $[39,40]$, and on the sample preparation technique [41].

Indeed, several works have been devoted to modeling the PA response of liquid-embedded nanoparticles, somehow accounting for a Tdependent $\alpha_{\text {wat }}$ and/or the presence of a TBC [8-11,14]. Calasso et al. [34] developed an analytical expression for the pressure field, under the assumption of heat deposited at a point in space. Prost and co-workers [35] numerically solved the PA problem for a gold NS immersed in water, taking into account the finite size of the NS. In their seminal work, they studied the effects of the pulse duration and of the NS's dimension on the PA signal, highlighting the contribution of non-linear effects.

Notwithstanding recent interesting works in the field

\footnotetext{
${ }^{1}$ The Grüneisen parameter is defined as $\Gamma=\alpha c^{2} / C$, where $\alpha$ is the volumetric expansion coefficient $\left(\mathrm{K}^{-1}\right), c$ is the speed of sound $(\mathrm{m} / \mathrm{s})$ and $C$ is the heat capacity per unit mass $(\mathrm{J} / \mathrm{kg} \mathrm{K})$ of the medium.
}

[21-22,42,43], modelling of the PA signal as a function of optical wavelength remains relatively unexplored. This work addresses the latter by investigating the PA signal generated by gold NS (representing the exogenous contrast agents) immersed in water (mimicking biological tissue) as a function of the exciting optical wavelength. The main goal of this work is to compare the PA signal - as a function of the optical wavelength - with the optical absorption coefficient, the latter measured in a standard optical transmission experiment and extracted from Lambert-Beer's law. In particular, we compare the wavelength dependence of the ratio between the PA signal contributions caused by water absorption and NS absorption, with the wavelength dependence of the ratio between the respective optical absorption coefficients as they would be measured in a standard optical transmission experiment, and extracted from Lambert-Beer's law. ${ }^{2}$ The results are based on an opto-thermo-acoustic model considering light absorption - across the visible and near-infrared (NIR) range - in the gold NS and in a surrounding water shell. The PA signal is triggered by the NP absorption at SPR whereas is essentially due to water absorption in the NIR. Given this focus, in the following we ignore effects that are not relevant for PA signal, such as optical scattering (which in any case is quite small, given the smallness of the NSs compared to the optical wavelengths in the NIR-blue range of interest). The analysis is performed over different NS sizes. Also the effect of the TBC is addressed. Specifically, a realistic value for the TBC is introduced and its effect on the PA generation is investigated for values of the laser pulse durations of $5 \mathrm{~ns}$ and $200 \mathrm{ps}$ respectively. The situation is compared against the one of a perfect thermal contact. ${ }^{3}$

The manuscript is organized as follows: in Section 2 we present the theoretical model, while Section 3 is devoted to the study of the dependence of the PA signal as a function of the optical wavelength in the linear regime. In Section 4 we analyze how the non-linearity affects the PA signal, while in Section 5 we compare the pressure waves generated by NSs of different sizes. Section 6 addresses the effect of the TBC on the generated PA signal.

\footnotetext{
${ }^{2}$ In this article, we assume that the optical absorption coefficient obtained from optical transmission measurements is not affected by optical scattering, e.g. by making use of integrating sphere measurements, which take scattering into account. As a matter of fact, one of the strong points of photoacoustic spectroscopy is that, contrary to a standard transmission measurement, its results are in principle not affected by optical scattering.

${ }^{3}$ In the perfect thermal contact scenario, the TBC between the gold and the water is assumed infinite.
} 


\section{Theoretical mode}

In this section we develop a model useful to interpret the properties of the optical wavelength dependent PA signal generated by an ensemble of metal NSs immersed in water. The model is based on the subsequent resolution of three physical problems: optics (to estimate light absorption in the system), thermics (to compute the temperature evolution in the system) and, finally, acoustics (to quanfity the photoacoustic signal).

In the present work we consider case studies satisfying the following assumptions: (i) the light penetration length is longer than the real sample's size, the spatial extension of the latter being $\sim 1 \mathrm{~mm}$; (ii) the interparticle distance is very large; (iii) the NSs have the same dimension. These hypotheses imply that (a) every NS has the same light absorption and generates the same pressure variations; (b) the pressure variations generated by every single NS can be computed neglecting the presence of the other NSs (i.e. optical and acoustic scattering due to the presence of the other NS is negligible). Hence, the photoacoustic signal generated by the real sample can be obtained by adding up the pressure variations computed considering one single NS immersed in water.

\subsection{Optics}

The first step in the modelling involves the quantification of the light absorption, which, in the case of metal NSs, displays a strong wavelength dependence. In particular, in the visible or NIR, light absorption may be maximized due to a resonance of the electronic oscillations in the metal NS, the so-called surface plasmon resonance (SPR) $[16,17,37,44]$. For this reason, by tuning the optical wavelength in the visible and NIR range, the dissipated optical energy in the NS can vary by more than one order of magnitude. For the sake of exemplification, in Fig. 2, we analyze the light absorption of a gold NS of radius $R_{s p h}=20 \mathrm{~nm}$ immersed in water. The blue curve (blue left axis) represents the absorption cross section ${ }^{4}\left(\sigma_{a b s, s p h}\right)$ as a function of wavelength (horizontal axis), showing a sharp peak (SPR) around $530 \mathrm{~nm}$ and a much lower absorption for higher wavelengths. The absorption cross section for the $5 \mathrm{~nm}$ and $100 \mathrm{~nm}$ radius gold NSs in water displays an analogous trend (we refer to Section 1 of Supplementary Material for further details concerning this point).

As far as water is concerned, its absorption coefficient $\beta_{\text {wat }}$ is also wavelength dependent and increases in the NIR region, as displayed in Fig. 2 red curve (right red axis). ${ }^{5}$ In the NIR part of the spectrum, light absorption by the water surrounding the NSs thus strongly contributes to the generated PA signal.

In order to account for the light absorption in water in our model, we ideally divide the real sample in small domains, each one containing just one NS. For the sake of simplicity, we assume that every domain is a sphere of radius $R_{\text {wat }}$, with the metal NS in the center. Given a spatially homogeneous NSs concentration $\rho_{N S}$, the external radius of the water domain reads $R_{\text {wat }}=\sqrt[3]{3 /\left(4 \pi \rho_{\mathrm{NS}}\right)}$. In other words, the small domain is a core-shell sphere, the NS being the core (see Fig. 1 panel b). Provided the light penetration depth is much longer than the sample thickness, the PA signal amplitudes generated by NSs located at different depth are the same. However, their arrival times at the detector are different. In acoustic imaging applications with a bandwidth of typically less than $10 \mathrm{MHz}$ and acoustic wavelengths and pixel sizes $\lambda=(1500 \mathrm{~m} / \mathrm{s}) / 10 \mathrm{MHz}=150 \mu \mathrm{m}$ or more, the detected low

\footnotetext{
${ }^{4}$ The absorption cross section of a NS quantifies the power dissipated in the NS and its unit has the dimension of an area. This quantity is defined as the ratio between the heat losses and the incident light intensity I (see Section 1 of Supplementary Material for further details).

${ }^{5}$ The water absorption coefficient displayed in Fig. 2 has been obtained as $\beta_{\text {wat }}=4 \pi k_{\text {wat }} / \lambda$, where $\lambda$ is the optical wavelength and $k_{w a t}$ is the imaginary part of the water refractive index. The values of $k_{\text {wat }}$ are taken from Ref. [45].
}

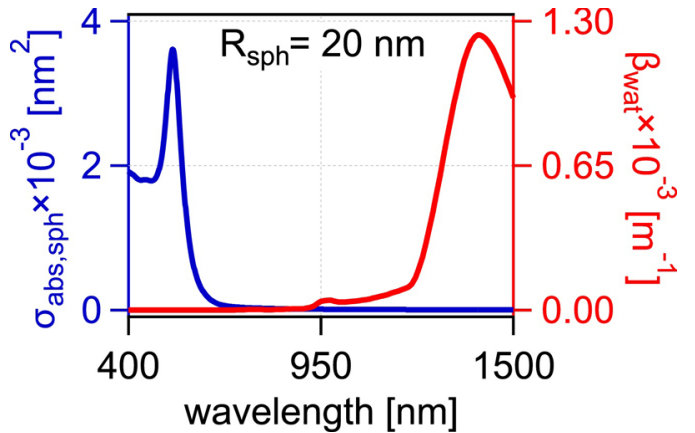

Fig. 2. Absorption cross section $\sigma_{a b s, s p h}$ of a $20 \mathrm{~nm}$ radius gold NS immersed in water (left axis, blue curve) and water absorption coefficient $\beta_{\text {wat }}$ (right axis, red curve) vs. optical wavelength (For interpretation of the references to color in this figure legend, the reader is referred to the web version of this article).

frequency components coming from different NSs within imaged pixels are coherently added up in phase. The PA signal amplitude and shape thus simply scales with the number of particles. Similarly, the low frequency components generated by water absorption coherently add up in phase with the ones generated by the NSs.

The dissipated optical power per unit volume is $\sigma_{a b s, s p h} I(t) / V_{s p h}$ in the metal core, where $V_{s p h}$ is the volume of the NS, and $\beta_{\text {wat }} I(t)$ in the water absorbing shell. ${ }^{6}$ Consistently with the above assumptions, the light intensity $I(t)\left(\mathrm{W} / \mathrm{m}^{2}\right)$ is assumed to be spatially homogeneous and gaussian in time:

$I(t)=2 \sqrt{\frac{\ln (2)}{\pi}} \frac{\Phi}{\tau} \exp \left\{-4 \ln (2)\left(\frac{t-t_{0}}{\tau}\right)^{2}\right\}$,

where $\tau$ is the pulse full-width-half-maximum (FWHM), $t_{0}$ is the pulse centre and $\Phi$ is the light fluence $\left(\mathrm{J} / \mathrm{m}^{2}\right)$. $^{7}$

\subsection{Thermics}

The dissipated laser power per unit volume, defined in Section 2.1, is introduced as a heat source term in the thermal diffusion equation.

The approach developed in Section 2.1 aims to calculate the pressure variations generated by the core-shell light absorbing domain and propagating towards the surroundings. For this reason, the thermal and acoustic problems are not restricted just to the core-shell light absorbing domain, but they are solved for the entire space.

Due to the radial symmetry of the problem, the physical quantities involved in the thermics can be expressed as a function of the radial coordinate $r$ only. The equations ruling the thermics - i.e. Fourier's law and the conservation of energy law respectively - hence read:

$\left\{\begin{array}{l}q_{r}=-k \frac{\partial T}{\partial r}, \\ \rho C \frac{\partial T}{\partial t}=-\frac{\partial q_{r}}{\partial r}-\frac{2}{r} q_{r}+Q(r, t),\end{array}\right.$

where $T(r)$ is the temperature, $q_{r}(r)$ the heat flux, $k(r)$ the thermal conductivity $(\mathrm{W} / \mathrm{m} \mathrm{K}), C(r)$ the heat capacity per unit mass $(\mathrm{J} / \mathrm{kg} \mathrm{K})$ and $\rho(r)$ the mass density. Consistent with the geometry of a metal NS immersed in an infinite domain of water, the latter three thermal

\footnotetext{
${ }^{6}$ In our calculations, the dissipated optical power per unit volume in the metal NS is assumed to be spatially homogeneous. The same consideration holds for the dissipated power per unit volume in the water absorbing shell.

${ }^{7}$ The constants appearing in expression (1) are necessary to assure that the time integral of the intensity gives exactly the fluence delivered to the system:
}

$\int_{-\infty}^{+\infty} I(t) \mathrm{dt}=\Phi$ 
parameters are described as step functions of the radial coordinate $r$, taking the metal (water) value for $0 \leq r \leq R_{s p h}$ (for $r>R_{s p h}$ ).

The dissipated power per unit volume reads:

$Q(r, t)= \begin{cases}\frac{\sigma_{\mathrm{abs}, \mathrm{sph}}}{V_{\mathrm{sph}}} I(t) & \text { for } 0 \leq r \leq R_{\mathrm{sph}}, \\ \beta_{\mathrm{wat}} I(t) & \text { for } R_{\mathrm{sph}}<r \leq R_{\mathrm{wat}}, \\ 0 & \text { for } r>R_{\mathrm{wat}} .\end{cases}$

The radial symmetry implies that at the origin $(r=0)$ the heat flux must vanish. This implies fulfilment of the boundary condition $q_{r}(r=0$, $t)=0$. At the interface between the metal NS and water, a thermal boundary conductance $\left(G_{T B C}\right)$ is assigned, resulting in the boundary conditions:

$\left\{\begin{array}{l}q_{r}\left(r=R_{\mathrm{sph}}^{-}, t\right)=q_{r}\left(r=R_{\mathrm{sph}}^{+}, t\right)=q_{r}\left(r=R_{\mathrm{sph}}, t\right), \\ T\left(r=R_{\mathrm{sph}}^{-}, t\right)-T\left(r=R_{\mathrm{sph}}^{+}, t\right)=\frac{1}{G_{\mathrm{TBC}}} q_{r}\left(r=R_{\mathrm{sph}}, t\right) .\end{array}\right.$

At time $t=0$ the system is assumed in equilibrium at a homogeneous temperature $T(r, t=0)=T_{0}=293.15 \mathrm{~K}$.

We have solved the thermal problem numerically with finite elements method (see Section 2 of Supplementary Material for details). For the sake of numerical resolution, it was necessary to limit the size of the computed domain. The water domain was truncated at a radius $r=R_{e n v}$, which was chosen to be large enough to avoid spurious boundary effects on the thermal dynamics. ${ }^{8}$

The solution of the thermal problem provides the temperature evolution at every spatial coordinate.

\subsection{Acoustics}

The obtained spatio-temporal temperature evolution is used to calculate the thermal strain, the latter triggering the pressure wave in water. The acoustic problem is accounted for in the frame of a linear elasticity model [35]. The equations system describing the acoustic problem reads:

$$
\left\{\begin{array}{l}
\frac{\partial v_{r}}{\partial t}=\frac{1}{\rho}\left[\frac{\partial \sigma_{\mathrm{rr}}}{\partial r}+\frac{2}{r}\left(\sigma_{\mathrm{rr}}-\sigma_{\theta \theta}\right)\right], \\
\frac{\partial \sigma_{\mathrm{rr}}}{\partial t}=(\lambda+2 \mu) \frac{\partial v_{r}}{\partial r}+2 \lambda \frac{v_{r}}{r}-\alpha(T)\left(\lambda+\frac{2}{3} \mu\right) \frac{\partial T}{\partial t}, \\
\frac{\partial \sigma_{\theta \theta}}{\partial t}=\lambda \frac{\partial v_{r}}{\partial r}+2(\lambda+\mu) \frac{v_{r}}{r}-\alpha(T)\left(\lambda+\frac{2}{3} \mu\right) \frac{\partial T}{\partial t}, \\
\sigma_{\phi \phi}=\sigma_{\theta \theta},
\end{array}\right.
$$

where $v_{r}$ is the radial component of the velocity field, $\sigma_{i j}$ are the components of the stress tensor and $T$ is the temperature (we refer to Section 3.1 of Supplementary Material for a rigorous derivation of System (5)). The appearing material properties - i.e. the mass density $\rho(r)$ and the two Lamé coefficients $\lambda(r)$ and $\mu(r)$ - are step functions of the radial coordinate $r$ and take the values of the metal for $0 \leq r \leq R_{s p h}$, and of water for $r>R_{s p h}$. The volumetric thermal expansion coefficient $\alpha$ is the one of the metal (whose temperature variations are neglected) for $0 \leq r \leq R_{s p h}$, and of the water for $r>R_{s p h}$. The thermal expansion coefficient of water has a rather strong dependence on temperature. Hence, the system of Eq. (5) becomes non-linear in temperature and, indirectly, in the optical fluence. Since, to a very good approximation, water is characterized by a null shear modulus $\mu$, the stress components in water read $\sigma_{r r}=\sigma_{\theta \theta}=\sigma_{\phi \phi}=-p$, where $p(r, t)$ is the pressure field in water, as described in Section 3.2 of Supplementary Material. Hence, by solving System (5) it is possible to access the pressure dynamics in

\footnotetext{
${ }^{8}$ For the sake of computational efficacy, we implemented an adiabatic boundary condition $q_{r}\left(r=R_{e n v}, t\right)=0$. However, since the computational domain is much bigger than the heat propagation space scale, the particular choice of the boundary condition at the external boundary is not relevant.
}

water, obtained as $p(r, t)=-\sigma_{r r}(r, t)$ for $r>R_{s p h}$.

At the interface between metal and water, the continuity of the radial stress $\sigma_{r r}$ and of the radial velocity $v_{r}$ is assumed. To preserve the spherical symmetry, we assign $v_{r}(r=0, t)=0$ at the origin, yielding $\left.\left.\frac{\partial v_{r}}{\partial r}\right|_{r=0, t} \sim \frac{v_{r}}{r}\right|_{r=0, t}$.

At the initial time $t=0$, the system is considered at rest. Hence, the initial values for the acoustic problem are $v_{r}(r, t=0)=0, \sigma_{r r}(r$, $t=0)=0, \sigma_{\theta \theta}(r, t=0)=0$ and $\sigma_{\phi \phi}(r, t=0)=0$.

We solve the acoustic problem with a finite difference scheme, following the approach proposed by Prost et al. [35]. As we did for the thermics, also for the acoustic problem we truncate the water domain at the coordinate $r=R_{e n v}$, with $R_{e n v}$ large enough to avoid unphysical reflection effects at the external boundary. ${ }^{9}$

\section{Photoacoustic effect in the linear regime}

\subsection{Temperature and pressure variations at $S P R$ and in the near-infrared}

In this section we report on results for the PA effect generated by a $20 \mathrm{~nm}$ radius NS surrounded by water. The linear case is considered in the current section, meaning that the water volumetric thermal expansion coefficient is assumed as constant $\alpha_{\text {wat }}=2.1 \times 10^{-4} \mathrm{~K}^{-1}$ [35].

The gold NS is considered so as to be consistent with the experiments reported in Ref. [30]. A summary of the thermal and mechanical properties of gold and water is reported in Table 1.

The thermal and acoustic response of the gold NS is calculated for the case of illumination with a laser pulse of fluence $\Phi=20 \mathrm{~J} / \mathrm{m}^{2}$ and temporal FWHM $\tau=5 \mathrm{~ns}$, values typically used for in PA measurements. In order to keep the entire light pulse within the simulation time domain, the temporal center of the pulse is located at time $t_{0}=10 \mathrm{~ns}$. A sketch of the temporal excitation evolution, normalized over its maximum value $\left(I(t) / I_{\max }\right)$, as a function of time is plotted in Fig. 3 a. A red dashed vertical line has been added to emphasize the time $t=t_{0}$ at which the laser intensity reaches its maximum.

Depending on the optical wavelength, the light absorption of water may give an important contribution to the total PA signal. To account for this fact, light absorption is also considered within a water shell surrounding the gold NS. The absorbing water shell external radius is chosen as $R_{w a t}=2.2 \mu \mathrm{m}$, corresponding to a NSs concentration of $\rho_{N S} \sim 3.8 \times 10^{-11}$ moles per liter, ${ }^{10}$ a value compatible with that achievable in real experimental situations [30].

In order to quantify the contribution of water light absorption to the total PA effect, we compute the temperature profile and the pressure variations for two limiting cases. First, we consider an optical wavelength falling within the range of the SPR, $\lambda=532 \mathrm{~nm}$, for which the light absorption within the gold NS is maximum while the water light absorption is very low (see Fig. 2). Next, we have considered the opposite scenario, that is we chose the wavelength in the NIR, $\lambda=1500 \mathrm{~nm}$, at which the water light absorption dominates. $\lambda=1500 \mathrm{~nm}$ wavelength bears great technological relevance, being the standard wavelength in telecom applications.

In Fig. 3b we depict the temperature variations profile $\Delta T=T(r$, $t$ ) $-T_{0}$ (color scale) for $\lambda=532 \mathrm{~nm}$, as a function of time (horizontal axis) and of the radial coordinate $r$ (vertical axis). The temporal and

\footnotetext{
${ }^{9}$ We assigned a rigid boundary condition $v_{r}\left(r=R_{\text {env }}, t\right)=0$. However, since for the investigated time span the computation domain is much larger than the spatial extent of the pressure wave propagation, the particular choice of the boundary condition at the external boundary is not relevant.

${ }^{10}$ In the wavelength range considered in this manuscript, i.e. $400-1500 \mathrm{~nm}$, the scattering cross section $\sigma_{\text {sca }}$ of a gold NS with radius not exceeding $100 \mathrm{~nm}$ is lower than $1.5 \times 10^{-13} \mathrm{~m}^{2}$. The effective absorption cross section of the light absorbing water shell is of the order of $\pi R_{\text {wat }}^{2} \sim 1.5 \times 10^{-11} \mathrm{~m}^{2}$, which is much bigger than $\sigma_{\text {sca }}$. As a consequence, the NS light scattering is negligible for all the case studies described in the manuscript.
} 
Table 1

Thermal and mechanical properties. The values were taken from Refs. $[35,37,38]$.

\begin{tabular}{lll}
\hline Material properties & Gold & Water \\
\hline Density $\left[\mathrm{kg} / \mathrm{m}^{3}\right]$ & $19.3 \times 10^{3}$ & $1 \times 10^{3}$ \\
Thermal conductivity [W/m K] & 318 & 0.6 \\
Heat Capacity [J/kg K] & 129 & $4.2 \times 10^{3}$ \\
First Lamé coefficient [GPa] & 147 & 2.25 \\
Second Lamé coefficient [GPa] & 27.8 & 0 \\
Volumetric thermal expansion coefficient $\left[\mathrm{K}^{-1}\right]$ & $0.43 \times 10^{-4}$ & $2.1 \times 10^{-4}$ \\
\hline
\end{tabular}

are significant only within a water shell thickness in the order of 20-30 $\mathrm{nm}$ around the NS.

The temperature profile reported in Fig. $3 \mathrm{~b}$ is used to calculate the pressure variations in liquid. Fig. 3e shows the pressure variations generated by the PA effect as a function of time, for a laser wavelength of $532 \mathrm{~nm}$. The pressure $p$ is evaluated far away from the NS, at a coordinate $r=10 \mu \mathrm{m}$. The dotted black curve represents the PA signal generated while accounting also for light absorption in water. On the other hand, the full orange line is obtained neglecting light absorption in water, i.e. setting $\beta_{\text {wat }}=0$. The dotted black and full orange curves

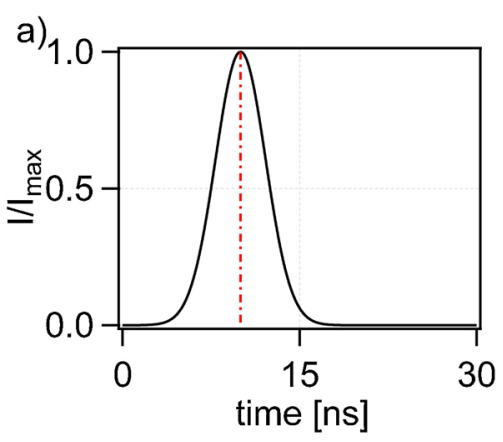

b)
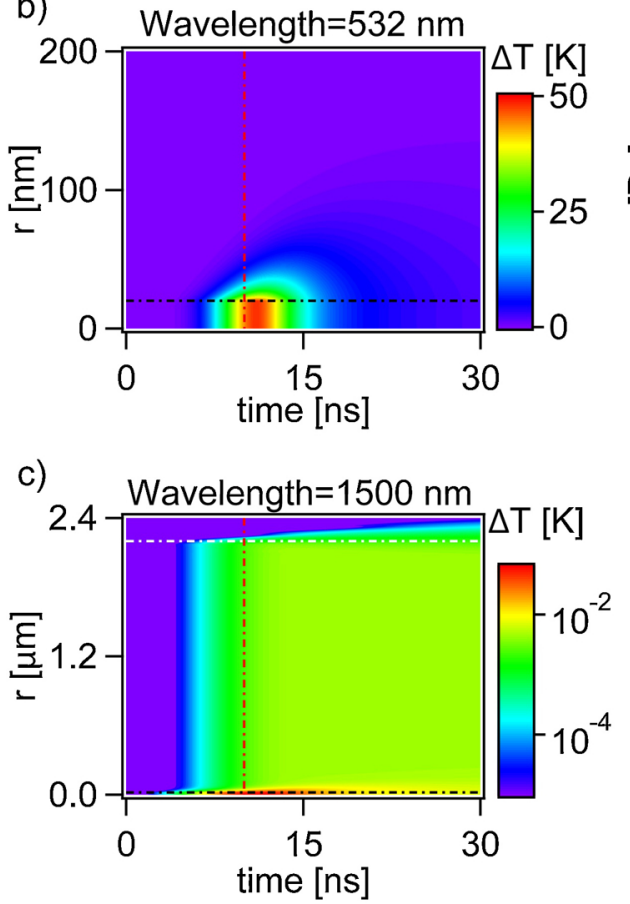
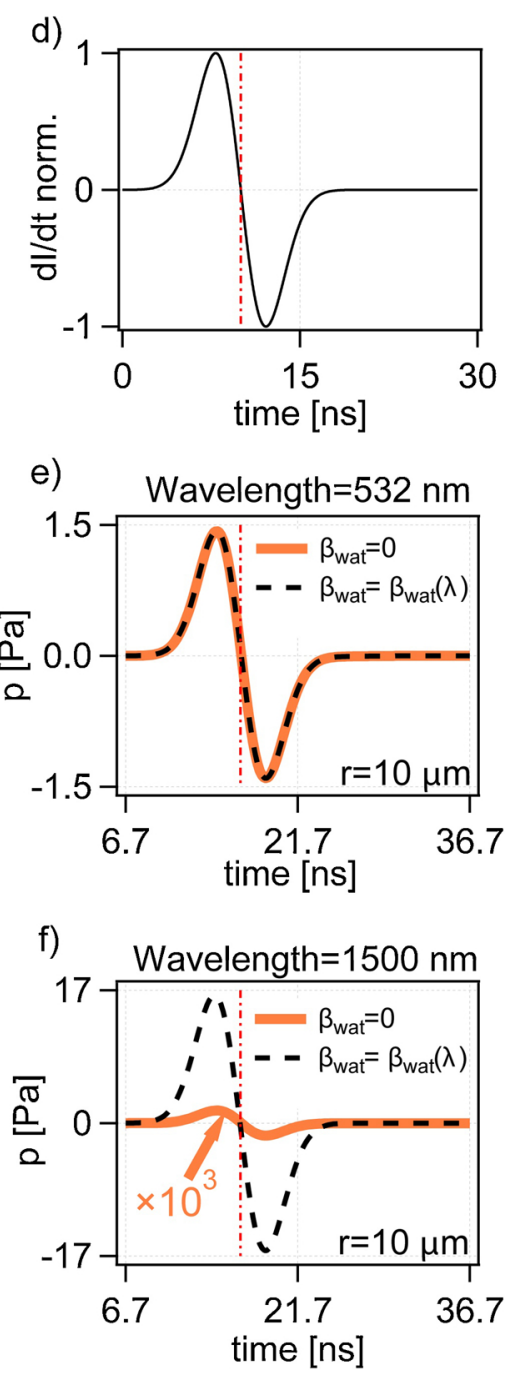

Fig. 3. (a) Plot of the temporal profile of the light intensity normalized at its maximum. (b) and (c) Temperature increase (color scale) as a function of the radial coordinate $r$ (vertical axis) and of time (horizontal axis). In panel b (panel c) the color scale is linear (log). The horizontal dashed black line represents the coordinate $r=R_{s p h}$, while the horizontal dashed white line in panel (c) represents the coordinate $r=R_{\text {wat }}$. Panels (e) and (f): plot of the PA pressure as a function of time, calculated at the spatial coordinate $r=10 \mu \mathrm{m}$. The dotted black (full orange) line is obtained considering (neglecting) light absorption in water. In panel $\mathrm{f}$ ), the PA trace obtained neglecting light absorption in water (orange curve) has been multiplied by a factor $10^{3}$. The wavelengths are $532 \mathrm{~nm}$ (panel b and e) and $1500 \mathrm{~nm}$ (panel c and f). (d) Time derivative of the light pulse temporal profile normalized at its maximum. A vertical dashed red line in panels (a) to (d) highlights the time instant maximizing the light intensity. The vertical dashed red line has been added to panels (e) and (f) to highlight the zero-crossing of the pressure curve. All panels refer to a $20 \mathrm{~nm}$ radius NS immersed in water, illuminated with a laser pulse of $5 \mathrm{~ns}$ FWHM and fluence $\Phi=20 \mathrm{~J} / \mathrm{m}^{2}$. Perfect thermal contact is assumed between the gold NS and the water (For interpretation of the references to color in this figure legend, the reader is referred to the web version of this article). radial coordinates have been limited to regions showing a significant temperature increase. Clearly, the maximum of temperature increase is $\sim 50 \mathrm{~K}$ and occurs in the NS shortly after the maximum intensity peak (compare panel a and panel $\mathrm{b}$, the vertical dashed red line being a guide for the eye). Furthermore, Fig. 3b shows that below the horizontal dashed black line (highlighting the coordinate $r=R_{s p h}$ ), the temperature depends only on the temporal coordinate, meaning that the temperature within the gold NS is spatially homogeneous. The maximum temperature increase at radial coordinates $r>150 \mathrm{~nm}$ is more than two orders of magnitude smaller than the maximum temperature increase $\Delta T_{\max }$ occurring in the NS and in the $20-30 \mathrm{~nm}$ thick shell of water surrounding it (exceeding $10 \mathrm{~K}$ ). These occurrences show that, for $\lambda=532 \mathrm{~nm}$, the temperature increase in water is mainly due to heat diffusion from the heated NS, rather than from direct heating of water due to electromagnetic absorption. The temperature variations in water coincide, confirming expectations that for this wavelength the light absorption of water is too small to generate a significant contribution to the PA pressure.

We have also verified that the PA signal is mainly generated by the water thermal expansion (as reported in Ref. [35]). We implemented a simulation forcing a null thermal expansion coefficient for the gold NS: the pressure variations recorded at $r=10 \mu \mathrm{m}$ displayed no relevant changes with respect to the results reported in Fig. 3e.

Taking the time derivative of Eq. (1) yelds:

$\frac{\mathrm{dI}}{\mathrm{dt}}=-16 \ln (2) \sqrt{\frac{\ln (2)}{\pi}} \frac{\Phi}{\tau^{3}}\left(t-t_{0}\right) \exp \left\{-4 \ln (2)\left(\frac{t-t_{0}}{\tau}\right)^{2}\right\}$.

By plotting $d I / d t$ normalized to its maximum (see Fig. 3d), we notice that the induced pressure variations (reported in panel e) follow the 
same profile, with a positive maximum followed by a negative minimum. This is consistent with the source term in the equation for the stress (and pressure) being proportional to the time derivative of the temperature, which in turn is solved considering a source term proportional to $I(t)$. The main frequency contributing to the PA signal is $\nu_{\max }=\sqrt{2 \ln 2} /(\pi \tau)=75 \mathrm{MHz}$, corresponding to the acoustic wavelength $\lambda_{\text {acoust }}=c_{\text {wat }} / \nu=20 \mu \mathrm{m}$ (we refer to Section 4 of Supplementary Material for further details).

It is important to note that, as elaborated above, the effective bandwidth of the PA signal in a typical detection configuration is further reduced to $10 \mathrm{MHz}$ or less, due to the low-pass characteristics of the piezoelectric or optic-elastic transducers.

Panel e reports the pressure calculated at $r=10 \mu \mathrm{m}$, obviously the PA signal arrives at a later time with respect to the absorption of the laser pulse. The time necessary for the pressure variations to propagate up to $r=10 \mu \mathrm{m}$ (calculated dividing the spatial coordinate by the speed of sound in water $c_{w a t}=1500 \mathrm{~m} / \mathrm{s}$ ) is $\sim 6.7 \mathrm{~ns}$. For this reason, in order to better appreciate the direct analogy between panels $\mathrm{d}$ and e, the horizontal scale in the latter has been set from $6.7 \mathrm{~ns}$ to $36.7 \mathrm{~ns}$ (exactly the same scale used in panel d, but shifted of $6.7 \mathrm{~ns}$ ).

In Fig. 3d the vertical dashed red line corresponds with the time instant $t=t_{0}$, and falls where the curve has a node.

Panels $c$ and $f$ of Fig. 3 are the analogies of panels $b$ and e for the case at $\lambda=1500 \mathrm{~nm}$, where light absorption from water is relevant indeed. In panel c a logarithmic color scale has been adopted.

Also in the present case, the maximum of the temperature occurs in the gold NS. The energy absorbed by the NS is confined in a very small volume, hence the dissipated power per unit volume is greater in the gold NS than in water. ${ }^{11}$

The maximum of the temperature increase in the NS is $\sim 50 \mathrm{mK}$, three orders of magnitude lower as compared to the case $\lambda=532 \mathrm{~nm}$, since $\sigma_{a b s, s p h}(\lambda=532 \mathrm{~nm}) / \sigma_{a b s, s p h}(\lambda=1500 \mathrm{~nm}) \sim 10^{3}$. Consistently, for excitation at $1500 \mathrm{~nm}$, the pressure signal magnitude obtained while neglecting light absorption in water is three orders of magnitude lower than the one obtained for excitation $\lambda=532 \mathrm{~nm}$ - compare the full orange curves in panel e and $\mathrm{f}$.

The situation is completely different once we include for the possibility of light absorption in water. For this case we obtain a minute temperature increase $(\sim 3 \mathrm{mK})$ throughout the entire water volume, hence, every portion of the volume yields a very tiny contribution to the PA effect. Nevertheless, the acoustic wavelength $\lambda_{\text {acoust }}$ is $\sim 10 R_{\text {wat }} \sim 22 \mu \mathrm{m}$, hence the pressure field contributed by each infinitesimal water volume within the unit cell, however small, add in phase. This results in a conspicuous total pressure variation, as depicted in Fig. 3f dotted black curve. According for light absorption in water, the total pressure variations for excitation at $\lambda=1500 \mathrm{~nm}$ exceed by an order of magnitude those obtained for excitation at $\lambda=532 \mathrm{~nm}$.

\subsection{Wavelength analysis}

In the current section we investigate how the PA signal varies with the optical wavelength and how it relates to the absorption coefficients. We run our model for several values of the optical wavelength and, for every computed case, we record the maximum of the pressure variations $p_{\max }$ evaluated at the spatial coordinate $r=10 \mu \mathrm{m}$. As elaborated above, due to bandwidth limitations of the laser pulse $(1 / \tau=200 \mathrm{MHz})$ and of typical PA imaging systems, to a good approximation, the shape of the PA signal is independent of the details of the generating entities, so that $p_{\max }$ is a suitable quantity to represent the strength of the overall PA signal. The dependence of $p_{\max }$ on the optical wavelength is plotted in Fig. 4a, solid black line. In order to appreciate the strong variations of $p_{\max }$, a logarithmic scale has been adopted on the vertical axis.

\footnotetext{
${ }^{11}$ The ratio between the dissipated power per unit volume in the NS and in the water at $1500 \mathrm{~nm}$ is $Q_{s p h}(t) / Q_{w a t}(t)=\sigma_{a b s, s p h} /\left(\beta_{w a t} V_{s p h}\right)=120$.
}
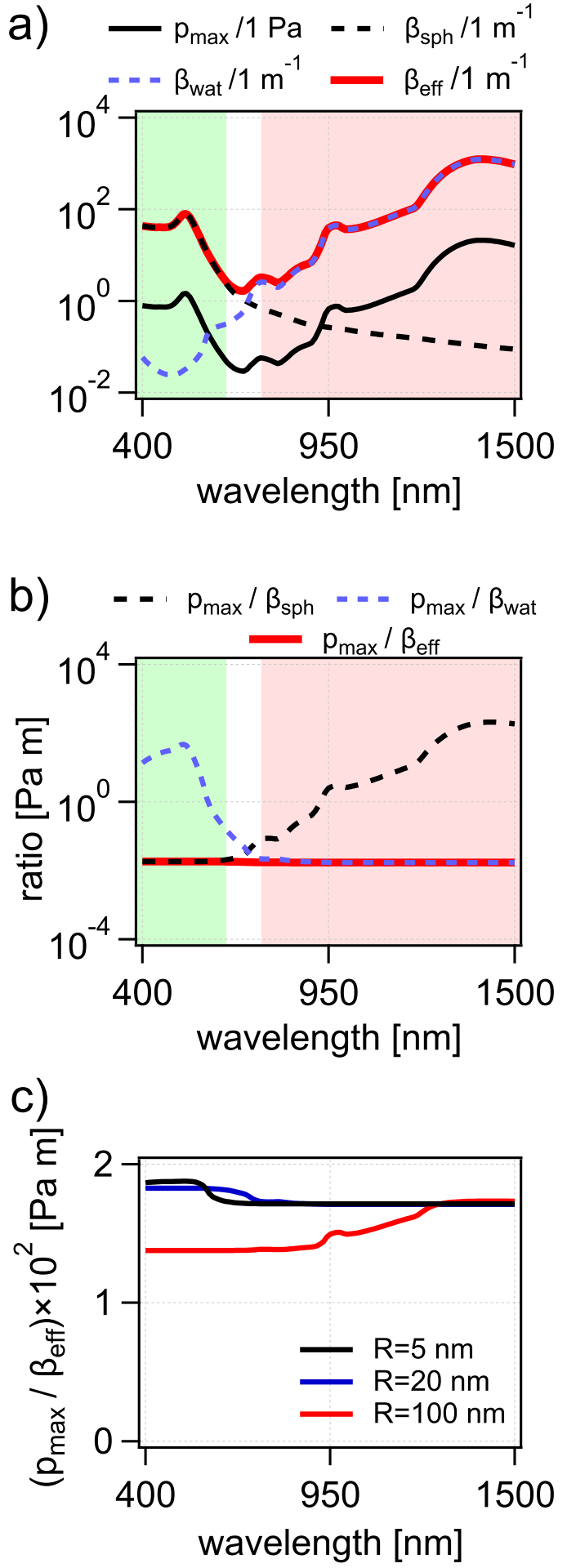

(caption on next page)

The highest PA signal is obtained in the NIR region, where water light absorption is the main source of the overall pressure variations. When $\lambda$ decreases from the NIR region to visible wavelengths, light absorption in water diminishes accordingly (see the red curve in Fig. 2). As a consequence, the PA signal drops by three orders of magnitude, reaching a minimum at $\lambda=700 \mathrm{~nm}$. Further lowering the wavelength, the PA signal increases again thanks to the strong light absorption in the gold NS, reaching a local maximum at $\lambda \sim 530 \mathrm{~nm}$ (SPR of the gold NS). In agreement with the results reported in Section 3.1, $p_{\max }$ at the local maximum $\lambda=530 \mathrm{~nm}$ is one order of magnitude lower than its value at 
Fig. 4. (a) Pressure maximum $p_{\max }$ recorded during all the acoustic dynamics time calculated at coordinate $r=10 \mu \mathrm{m}$ (full black line), NSs effective absorption coefficient $\beta_{\text {sph }}$ (dashed black line), water absorption coefficient $\beta_{\text {wat }}$ (dashed blue line) and total effective absorption coefficient $\beta_{\text {eff }}$ (full red line) as functions of the optical wavelength. (b) Ratio $p_{\max } / \beta_{s p h}$ (dashed black line), $p_{\max } / \beta_{\text {wat }}$ (dashed blue line) and $p_{\max } / \beta_{\text {eff }}$ (full red line), as functions of the optical wavelength. In both panels, the region where the particle absorption coefficient dominates the water absorption coefficient is shaded in light green, while the region where $\beta_{\text {wat }}$ dominates is shaded in light red. The region where $\beta_{\text {sph }}$ and $\beta_{\text {wat }}$ are comparable is left blank. In both panels, the vertical axis is in log scale. Both panels refer to a $20 \mathrm{~nm}$ radius NS immersed in water, illuminated by a laser pulse of $5 \mathrm{~ns}$ FWHM and fluence $\Phi=20 \mathrm{~J} / \mathrm{m}^{2}$. (c) Ratio $p_{\max } /$ $\beta_{\text {eff }}$ for different NS diameters ( $5 \mathrm{~nm}$ (black), $20 \mathrm{~nm}$ (blue) and $100 \mathrm{~nm}$ (red) (For interpretation of the references to color in this figure legend, the reader is referred to the web version of this article).

\section{$\lambda=1500 \mathrm{~nm}$}

In the context of most optical transmission measurements, a biological tissue embedding exogenous contrast agents may be considered as an effective homogeneous medium, characterized by an effective ab-

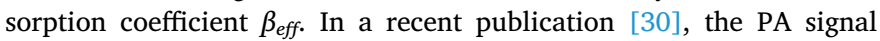
generated by gold nanorods immersed in water has been investigated. The measured absorption coefficient and the generated PA signal, as functions of the excitation optical wavelength, were compared and an unexpected lack of proportionality between them was recorded in the wavelength range 700-900 $\mathrm{nm}$.

For this reason, here we calculate the absorption coefficient of the effective medium $\beta_{\text {eff }}$, the latter being composed by $20 \mathrm{~nm}$ radius gold NSs dispersed in water. In the regime of low light absorption and small NSs, the expression for $\beta_{\text {eff }}$ reads (see Section 5 of Supplementary Material for the derivation):

$\beta_{\mathrm{eff}}(\lambda)=\beta_{\mathrm{sph}}(\lambda)+\beta_{\mathrm{wat}}(\lambda)$

where $\beta_{\text {sph }}(\lambda)=\sigma_{a b s, s p h}(\lambda) / V$ is the absorption coefficient of the effective medium when light absorption in water is switched off (i.e. the only absorbing elements are the gold NSs) and $V$ is the volume of the light absorbing water domain.

In Fig. $4 \mathrm{a} \beta_{\text {eff }}$ is plotted as a function of wavelength (full red curve) in logarithmic scale. The separate contributions to the optical absorption, i.e. that of the NS $\beta_{\text {sph }}$ (dashed black line) and that of water $\beta_{w a t}$ (dashed blue line) are shown as well.

For $\lambda<640 \mathrm{~nm}$, at the SPR, light absorption in the gold NS dominates: $\beta_{\text {eff }} \sim \beta_{\text {sph }}$ (green shaded region in Fig. 4). For $\lambda>750$ water light absorption prevails: $\beta_{\text {eff }} \sim \beta_{\text {wat }}$ (pink shaded region in Fig. 4). ${ }^{12}$ In the wavelength range $640 \mathrm{~nm}<\lambda<750 \mathrm{~nm}$ (left blank in Fig. 4), $\beta_{\text {sph }}$ and $\beta_{s p h}$ are of the same order of magnitude, hence they both contribute significantly to $\beta_{\text {eff. }}$.

Fig. 4 a suggests that $p_{\max }$ (full black line) and $\beta_{\text {eff }}$ (full red line) could be proportional. This seems to be confirmed in Fig. 4b, which shows the ratio $p_{\max } / \beta_{\text {eff }}$ (full red line) as a function of wavelength. Looking more closely on a linear scale (Fig. 4c), it turns out that for the $20 \mathrm{~nm} \mathrm{NS}$, and for a $5 \mathrm{~nm} \mathrm{NS}$, below $800 \mathrm{~nm}$, where the plasmon resonance of the NS starts to play, the ratio slightly increases, of about $8 \%$. For a large NS of $100 \mathrm{~nm}$, below $800 \mathrm{~nm}$, the ratio decreases of about $20 \%$. These moderate but nevertheless different trends between different NS diameters are discussed further in Section Section 6 of Supplementary Material.

\footnotetext{
${ }^{12}$ In the NIR, $\beta_{s p h}=\sigma_{a b s, s p h} / V$ is negligible because of the small value of $\sigma_{a b s, s p h}$, whereas $\beta_{\text {wat }}$ prevails: $\beta_{\text {sph }}<\beta_{\text {wat }}$ (see Fig. 4). On the other hand, the expression of the power dissipated per unit volume $Q$ is bigger in the gold NS (where $Q_{s p h} \propto \sigma_{a b s, s p h} / V_{s p h}$ ) than in the surrounding water (where $Q_{w a t} \propto \beta_{w a t}$ ). Hence, the overall energy absorbed by the NS is much smaller than that absorbed by the surrounding water, but the opposite holds true for the absorbed energy density. For this reason, also in the NIR region, the maximum temperature is recorded within the NS, as described in Section 3.1.
}

For the sake of completeness, in Fig. 4b we display also the ratio $p_{\max } / \beta_{\text {sph }}$ (dashed black line) and $p_{\max } / \beta_{\text {wat }}$ (dashed blue line), the latter two quantities being constant and superposed to $p_{\max } / \beta_{\text {eff }}$ in the wavelength ranges where $\beta_{\text {sph }}$ or $\beta_{\text {wat }}$ dominate, respectively.

\section{Photoacoustic effect in the non-linear regime}

The simplification adopted in Section 3, where the temperature dependence of the water expansion coefficient - i.e. non-linearity - was neglected, proved very useful to understand the mechanisms of PA signal generation when light absorption in water is considered. However, as reported in Fig. 3b, the temperature increase in water can easily reach $40 \mathrm{~K}$ even upon moderate light fluence, evoking the emergence of detectable non-linear effects. Hence, the current section is devoted to the study of non-linear contributions. In analogy with the previous sections, a gold NS of $20 \mathrm{~nm}$ radius illuminated with a pulse with $\tau=5 \mathrm{~ns}$ and $\Phi=20 \mathrm{~J} / \mathrm{m}^{2}$ is considered.

The non-linearity is based on the strong temperature dependence of the water volumetric thermal expansion coefficient $\alpha_{\text {wat }}$. In Fig. 5 we display the values of $\alpha_{w a t}$ as a function of temperature.

In order to appreciate the non-linearity, the temperature in water has to increase considerably. However, as we have seen in Fig. 3b and c, the temperature variations within the system strongly depend on the optical wavelength, decreasing by three orders of magnitude upon wavelength variation from $532 \mathrm{~nm}$ to $1500 \mathrm{~nm}$. As a consequence, a systematic study of the non-linear effects as a function of the optical wavelength is required for a full comprehension of the PA effect in the real situation.

Fig. $3 b$ and $c$ indicate that for $\lambda \leq 1500 \mathrm{~nm}$, the maximum of the temperature increase is located in the gold NS. Hence, the maximum temperature increase in the water near the gold NS can be considered as the upper limit of the temperature increase throughout the entire water domain. Fig. 6 panel a shows the maximum (over all the computed times) temperature increase $\Delta T_{\max }$ recorded in water just outside the gold NS ( $r=R_{s p h}+2 \mathrm{~nm}^{13}$ ) as a function of the optical wavelength. $\Delta T_{\max }$ exceeds $1 \mathrm{~K}$ only for wavelengths in proximity of the SPR, i.e. $\lambda<600 \mathrm{~nm}$. As a consequence, non-linear effects may be important only around the SPR.

To prove the latter assertion, we compute the PA trace at $\lambda=532 \mathrm{~nm}$, where $\Delta T_{\max }$ just outside the gold NS reaches $40 \mathrm{~K}$ (as highlighted by the vertical dashed red line in Fig. 6a). The time evolution of the corresponding pressure variations (calculated at the coordinate $r=10 \mu \mathrm{m}$ ) is plotted in Fig. 6 panel b. The dotted black curve represents the PA signal obtained considering the non-linearity (using $\alpha_{\text {wat }}$ reported in Fig. 5). For the sake of comparison, also the pressure variations in the linear regime (full orange line), i.e. keeping $\alpha_{\text {wat }}=2.1 \times 10^{-4} \mathrm{~K}^{-1}$ fixed, are shown. The latter curve corresponds to the PA signal already displayed in Fig. 3e. The non-linearity increases the amplitude of the PA effect. In particular, the minimum of $p$ reported in panel $b$ changes from -1.4 (linear case) to -2.5 (non-linear case), with an amplitude increase of $\sim 80 \%$. At the maximum, the amplitude increases by more than $50 \%$.

The situation is different when the optical wavelength is out of the SPR. For instance, Fig. $6 \mathrm{c}$ shows the PA signal obtained using $\lambda=750 \mathrm{~nm}$. Since $\Delta T_{\max }$ in water near the NS's surface is now $\sim 0.4 \mathrm{~K}$ (as emphasized by the dashed blue line in Fig. 6a), $\alpha_{\text {wat }}$ does not vary significantly from the equilibrium value $\alpha_{\text {wat }}=2.1 \times 10^{-4} \mathrm{~K}^{-1}$ and the non-linearity is suppressed (the dotted black and full orange curves in panel $\mathrm{c}$ are superposed).

In order to get further insight on the effect of the non-linearity, in Fig. 8 we report the maximum of the pressure variations as a function of

\footnotetext{
${ }^{13}$ The evaluation of the water maximum temperature is performed $2 \mathrm{~nm}$ away from the gold NS's surface to avoid numerical errors due to material discontinuity at $r=R_{s p h}$.
} 


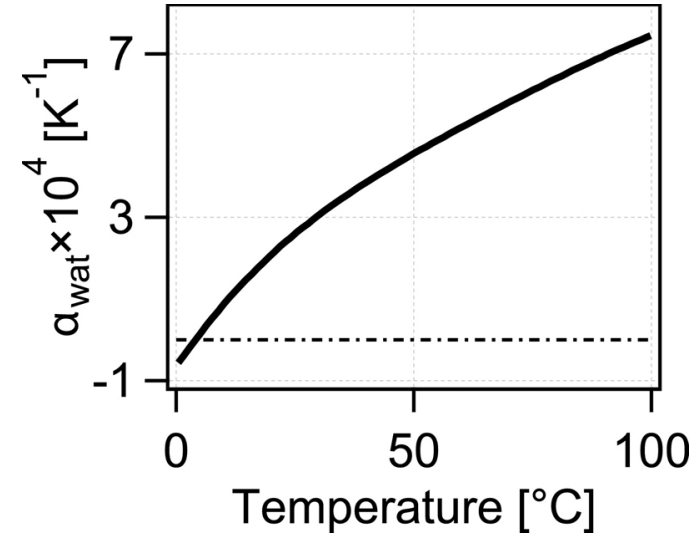

Fig. 5. Volumetric expansion coefficient of water $\alpha_{w a t}$ as a function of temperature (data taken from [35]). The horizontal dashed-dotted black line highlights the coordinate at which $\alpha_{\text {wat }}=0$.

the wavelength, both in the linear regime (full red line), and when including the non-linearity (dashed-dotted blue line). For $\lambda>600 \mathrm{~nm}$ the two curves are superposed, indicating that the non-linearity is negligible. On the other hand, at the SPR, non-linearity gives an important contribution, increasing the amplitude of $p_{\max }$ by more than $50 \%$ (mind the fact that in Fig. 8 the log scale is applied on the vertical axis).

In Fig. 7 we report the ratio between the pressure maximum $p_{\max }$ (recorded during all the acoustic dynamics time calculated at coordinate $r=10 \mu \mathrm{m}$ ) and $\beta_{e f f}$ as a function of wavelength. The dashed black line is obtained when the non-linearity is accounted for. For the sake of comparison, we report the same ratio obtained when the nonlinearity is neglected (full red line), already shown in Fig. 4.

When non-linearity is included, the ratio is not constant for wavelengths in the SPR, that is where the temperature increase is important. On the other hand, for wavelengths out of the SPR (where the temperature increase is very mild), the ratios $p_{\max } / \beta_{\text {eff }}$ obtained including or neglecting the non-linearity are flat and perfectly superposed. Hence, the proportionality between the photoacoustic signal and $\beta_{\text {eff }}$ does hold as long as the non-linear effects are absent. On the other hand, when the non-linearity is important, the proportionality between the photoacoustic signal and $\beta_{\text {eff }}$ is violated. The latter result indicates that the lack of proportionality between the PA signal and the absorption coefficient, reported for wavelengths not in the SPR in Ref. [30], is necessarily not ascribable to aspects of the combined light absorption in water and in the gold nanorods.

The main result of the current section is that upon light illumination with a moderate fluence $\left(\Phi=20 \mathrm{~J} / \mathrm{m}^{2}\right.$ ), non-linearity effects on the PA signals can be enhanced or suppressed by tuning the optical wavelength, with significant effects on the amplitude of the generated pressure waves.

\section{Influence of the size of the nanosphere on the photoacoustic signal}

In the current section we analyze how the size of the gold NS affects the PA signal. In the previous sections, we studied the case of a gold NS of radius $20 \mathrm{~nm}$. In the following, we compare the PA signal with the ones of a smaller NS of radius $5 \mathrm{~nm}$ and a bigger one of radius $100 \mathrm{~nm}$. The wavelength dependences for the 3 cases are compared in Fig. 8, for both a non-linear scenario, taking into account the temperature dependence of the thermal expansion coefficient, and an artificial linear scenario, fixing the thermal expansion coefficient to a temperature independent value $\alpha_{\text {wat }}=2.1 \times 10^{-4} \mathrm{~K}^{-1}$.

All the curves are superposed for wavelengths in the NIR. This is due to the fact that for long wavelengths, light absorption by water is the
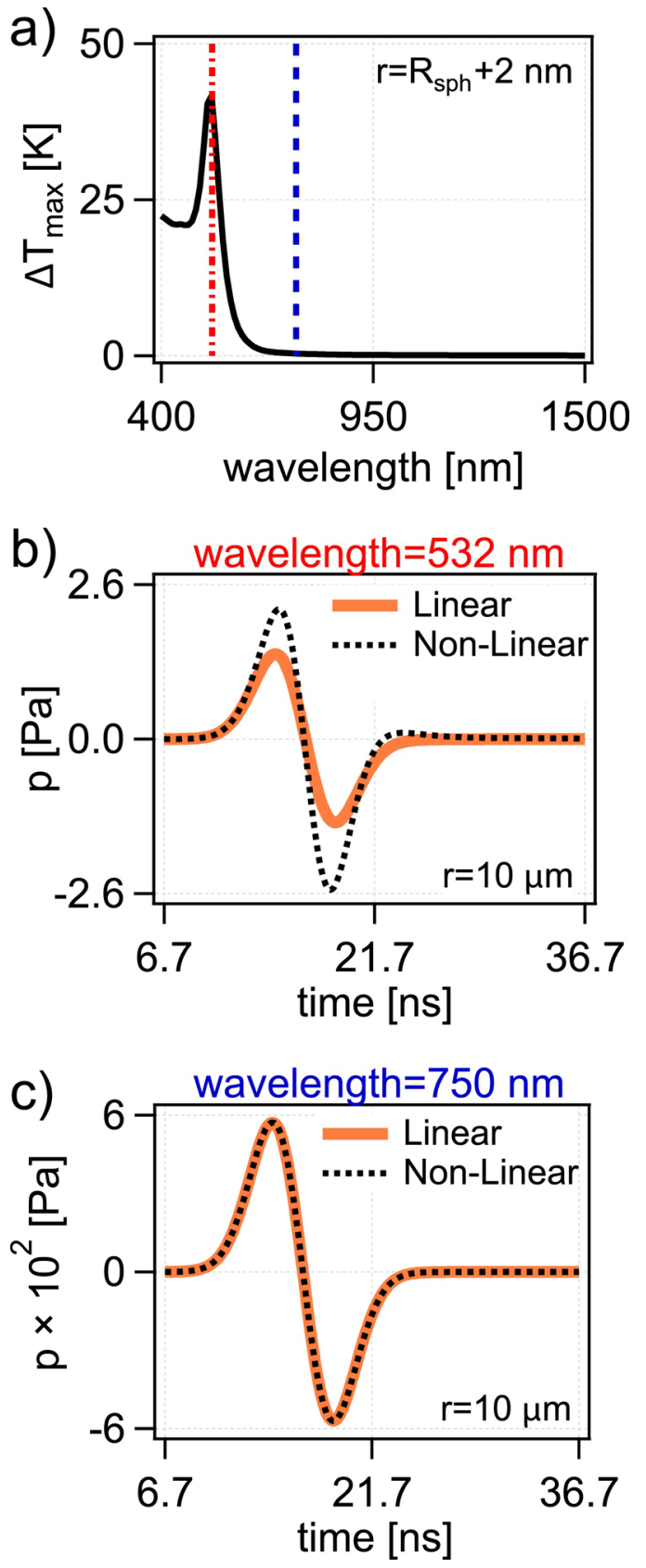

Fig. 6. (a) Maximum temperature increase $\Delta T_{\max }$ recorded throughout the thermal dynamics at the coordinate $r=R_{s p h}+2 \mathrm{~nm}$ as a function of the wavelength of the laser pulse. (b) and (c) plot of the pressure variations (vertical axis) as function of time (horizontal axis) calculated at the spatial coordinate $r=10 \mu \mathrm{m}$. The dotted black (full orange) line is obtained by taking into account (neglecting) the temperature dependence of the water expansion coefficient, i.e. considering non-linear (only linear) effects in the PA signal. The optical wavelength in panel b (panel c) is $532 \mathrm{~nm}(750 \mathrm{~nm})$. The optical wavelengths used in panel $\mathrm{b}$ and $\mathrm{c}$ have been highlighted in panel a with a dashed vertical red and blue line, respectively. All the panels refer to a $20 \mathrm{~nm}$ radius NS embedded in water, illuminated with a laser pulse of $5 \mathrm{~ns}$ FWHM and fluence $\Phi=20 \mathrm{~J} / \mathrm{m}^{2}$ (For interpretation of the references to color in this figure legend, the reader is referred to the web version of this article).

main mechanism generating the PA signal. Hence, in this regime, the presence of the NSs and their size is not relevant.

The situation changes for shorter wavelengths, for which the PA signal is mainly generated by the absorption of the NS. In particular, for $\lambda<700 \mathrm{~nm}, p_{\max }$ increases with increasing NS diameter. We refer to Section 6 of Supplementary Material for further details. 


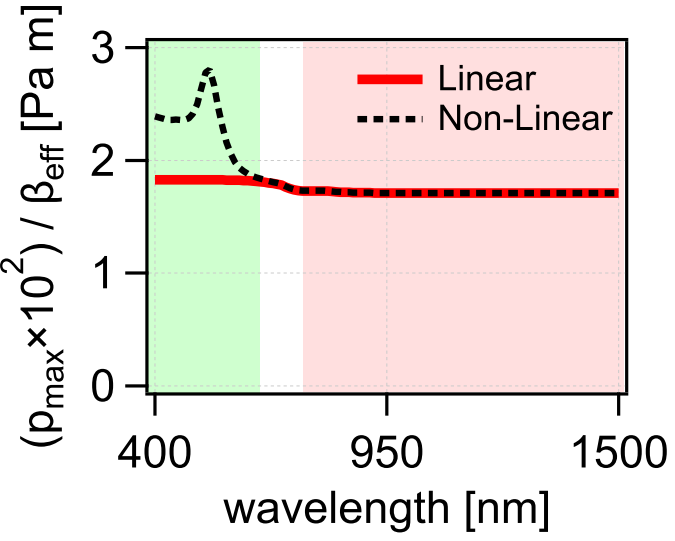

Fig. 7. Optical wavelength dependence of the PA signal divided by the effective optical absorption coefficient $p_{\max } / \beta_{\text {eff }}$ of a $20 \mathrm{~nm}$ NS illuminated by a laser pulse of $5 \mathrm{~ns}$ FWHM and fluence $\Phi=20 \mathrm{~J} / \mathrm{m}^{2}$. The pressure maximum $p_{\max }$, recorded during all the acoustic dynamics time calculated at coordinate $r=10 \mu \mathrm{m}$, is evaluated including (dashed black line) or neglecting (full red line) the non-linearity. In analogy with Fig. 4, regions where the light absorption in the NS and in the water dominate are shaded in light green and red, respectively (For interpretation of the references to color in this figure legend, the reader is referred to the web version of this article).

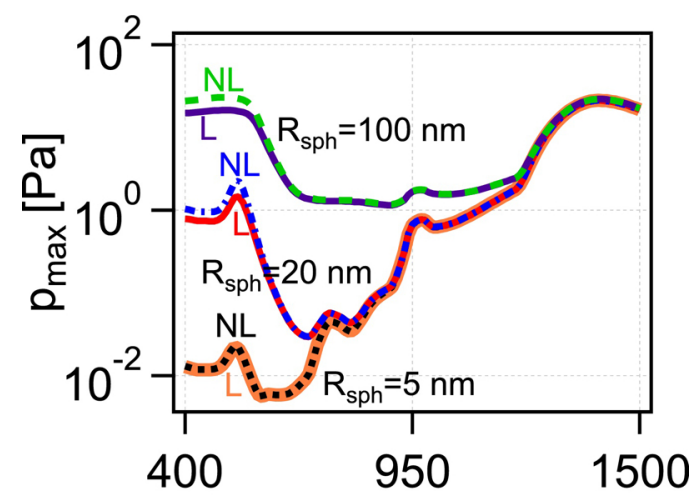

\section{wavelength $[\mathrm{nm}]$}

Fig. 8. Plot of the pressure maximum $p_{\max }$ (vertical axis, log scale) recorded during the entire acoustic dynamics at the coordinate $r=10 \mu \mathrm{m}$ as a function of the optical wavelength (horizontal axis). The dotted black (full orange) line corresponds to the non-linear (linear) PA spectrum for a NS of radius $5 \mathrm{~nm}$, the dashed-dotted blue (full red) line corresponds to the non-linear (linear) PA spectrum for a NS of radius $20 \mathrm{~nm}$, while the dashed green (full dark purple) line corresponds to the non-linear, NL (linear, L) PA spectrum for a NS of radius $100 \mathrm{~nm}$. In the investigated scenario, a light pulse of $5 \mathrm{~ns}$ FWHM and fluence $\Phi=20 \mathrm{~J} / \mathrm{m}^{2}$ is assumed (For interpretation of the references to color in this figure legend, the reader is referred to the web version of this article).

As for the non-linearity effect when working with a wavelength close to the SPR, for the considered case, the temperature increase in the smallest NS is smaller than $4 \mathrm{~K}$, hence non-linearity hardly plays.

Nonlinear signal enhancement does become observable for the two larger NSs, where, due to the larger optical absorption cross section at the SPR, the temperature increase exceeds $30 \mathrm{~K}$. Combining the influencing aspects of absorption cross section, heat distribution, signal generation efficiency and signal generating shell volume, the non-linear signal enhancement increases with increasing particle diameter (see also Supplementary Materials, Section 6).

\section{Influence of the thermal boundary resistance}

In the previous sections, for the sake of simplicity, we assumed perfect thermal contact between the gold NS and water. In the following, we investigate the changes in the computed PA signal when a realistic value of $G_{T B C}$ is assigned.

We now consider a gold NS of radius $20 \mathrm{~nm}$, excited with a laser pulse at wavelength $532 \mathrm{~nm}$, fluence $\Phi=20 \mathrm{~J} / \mathrm{m}^{2}$ and time duration $\tau=5 \mathrm{~ns}$ at FWHM. Fig. 9 panel a shows the temperature increase calculated at the spatial coordinate $r=R_{s p h}+2 \mathrm{~nm}$. Panel b shows the pressure variations calculated at the spatial coordinate $r=10 \mu \mathrm{m}$. The dotted black line corresponds to the case of perfect thermal contact (for sake of computation, perfect thermal contact is mimicked with an irrealistically large TBC value, i.e. $G_{T B C}=1 \times 10^{20} \mathrm{~W} / \mathrm{m}^{2} \mathrm{~K}$ ), while the full orange line was obtained for $G_{T B C}=1.05 \times 10^{8} \mathrm{~W} / \mathrm{m}^{2} \mathrm{~K}$ [38]. Both in panel $a$ and panel $b$ the two curves are superposed, meaning that the assumption of a perfect thermal contact is, for the given laser parameters and NP size, a good approximation of the real situation. We can rationalize this result in the frame of a lumped element model. The heat flowing out of the NS encounters two thermal resistances in series $\Omega_{T B C}$ and $\Omega_{w a t} ; \Omega_{T B C}=1 / G_{T B C}$ is the thermal boundary resistance and $\Omega_{\text {wat }}=\sqrt{\gamma_{\text {wat }} \tau} / k_{\text {wat }}$ is the thermal resistance of the heated water portion during the pulse temporal duration, $\gamma_{w a t}=k_{\text {wat }} /\left(\rho_{\text {wat }} C_{w a t}\right)$ being the thermal diffusivity in water. The latter may be understood realizing that $\sqrt{\gamma_{\text {wat }} \tau}$ is of the order of the distance covered by heat during the pulse duration and hence $k_{\text {wat }} / \sqrt{\gamma_{\text {wat }} \tau}$ is the thermal conductance of the heated portion of the liquid. The resistance values are reported in Table 2, whereas $\Omega_{T B C}=0$ for the perfect thermal contact case. For $\tau=5 \mathrm{~ns}$ we obtain $\Omega_{\text {wat }} \gg \Omega_{T B C}$, hence $\Omega_{T B C}$ can be disregarded.

When the pulse duration is reduced, $\Omega_{\text {wat }}$ decreases accordingly. For instance, for the case of $\tau=200 \mathrm{ps}, \Omega_{w a t} \sim \Omega_{T B C}$. In this case, the TBC has to be accounted for. This consideration agrees with the plots of the time evolution of $\Delta T$ and $p$ reported in Fig. 9c and d, where the curves obtained using the perfect thermal contact (dotted black line) and the realistic value of TBC (full orange line) differ. The presence of the TBC slows heat diffusion out of the NS, resulting in a reduced PA signal.

The conclusion that, for long enough laser pulses, the TBC does not alter the thermal dynamics in water is consistent with the expression for the water temperature obtained upon steady state illumination (see Section 7 of Supplementary Material). Consequently, in the latter scenario the generated PA signal is not altered by the value of the TBC.

We now give an estimate of the laser pulse duration, $\tau_{c}$, above which the effect of TBC on the PA signal may be neglected. The PA signal depends on the water temperature and the latter, within the lumped element model, is not affected by the TBC provided $\Omega_{w a t} \gg \Omega_{T B C}$, yielding $\tau \gg \tau_{c}=k_{\mathrm{wat}}^{2} /\left(\gamma_{\mathrm{wat}} G_{\mathrm{TBC}}^{2}\right)$. In the present case, $\tau_{c}$ is $230 \mathrm{ps}$.

If one were to reduce $G_{T B C}$ by an order of magnitude, for instance by functionalizing the NS surface or coating it with an insulating layer, the 5 ns laser pulse would fall short of $\tau_{c}$, leading to a significant change in the PA signal.

\section{Conclusions}

We theoretically investigated the photoacoustic (PA) effect generated by a gold nanosphere (NS) immersed in water, as a function of the optical wavelength of the laser pulse. The photoacoustic signal is computed with an opto-thermo-mechanical model, the latter considering light absorption in a NS and in the surrounding water shell.

Simulation results show that, for the case of PA excitation of a $20 \mathrm{~nm}$ radius NS with optical wavelengths tuned around the surface plasmon resonance ( $500-600 \mathrm{~nm}$ ), the energy is mostly absorbed by the NS, leading to a temperature increase in the surrounding water up to $\sim 50 \mathrm{~K}$ upon moderate light fluence. The launched pressure wave mainly results from the thermal expansion of the water; the contribution of the thermal expansion in the NS is negligible. In the NIR spectral range, the generation of the PA signal is ruled by water light absorption. In the latter case, the temperature increase is less than $1 \mathrm{~K}$. However, the pressure variations, being generated in a large volume of water, leads to a quite large PA signal. 

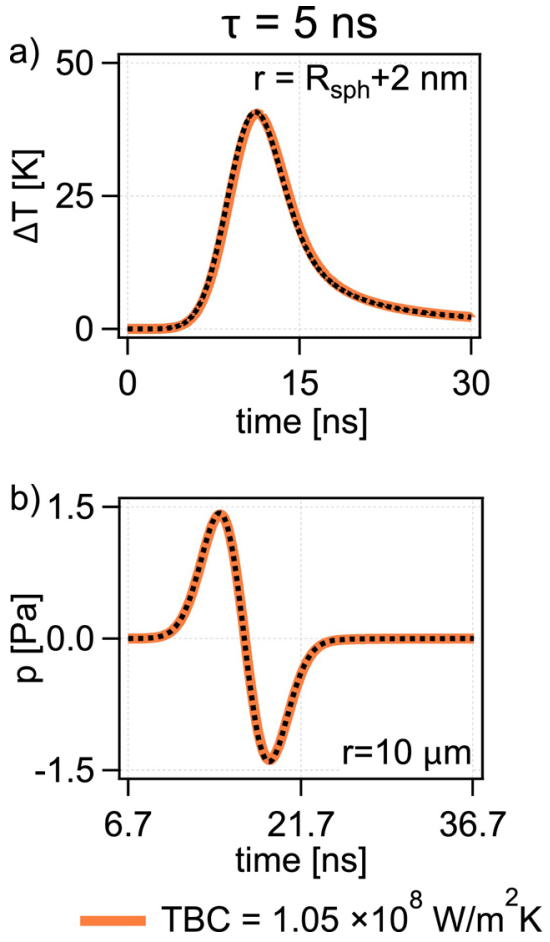
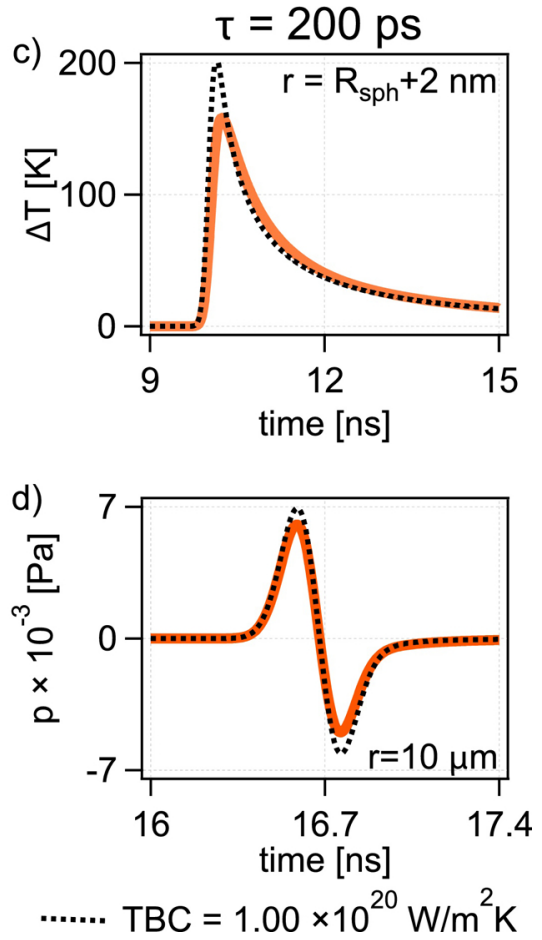

Fig. 9. Panel a and c: Plot of the temperature increase $\Delta T$ as a function of time, calculated at the coordinate $r=R_{\text {sph }}+2 \mathrm{~nm}$. Panel b and d: Plot of the pressure variations as a function of time, calculated at the spatial coordinate $r=10 \mu \mathrm{m}$. The dotted black lines have been calculated assuming perfect thermal contact between the gold NS and the water (i.e. $G_{T B C}=1 \times 10^{20} \mathrm{~W} / \mathrm{m}^{2} \mathrm{~K}$, mimicking an infinite TBC), while the full orange lines have been calculated for the actual value of TBC between gold and water (i.e. $G_{T B C}=1.05 \times 10^{8} \mathrm{~W} / \mathrm{m}^{2} \mathrm{~K}$ [38]). All calculations have been performed for the case of a NS of $20 \mathrm{~nm}$ radius illuminated by a light pulse of wavelength $532 \mathrm{~nm}$ and fluence $\Phi=20 \mathrm{~J} / \mathrm{m}^{2}$. In panel a and $\mathrm{b}$ $\tau=5 \mathrm{~ns}$, while in panel $\mathrm{c}$ and $\mathrm{d} \tau=200 \mathrm{ps}$ (For interpretation of the references to color in the text, the reader is referred to the web version of this article).
Table 2

First column: $\Omega_{T B C}=1 / G_{T B C}$ for the case of real TBC between gold and water. Second and third column: $\Omega_{\text {wat }}=\sqrt{\gamma_{\text {wat }} \tau} / k_{\text {wat }}$ for a pulse with $\tau=5$ ns and $\tau=200 \mathrm{ps}$, respectively.

\begin{tabular}{lll}
\hline$\Omega_{T B C}$ & $\Omega_{\text {wat }}$ for $\tau=5 \mathrm{~ns}$ & $\Omega_{\text {wat }}$ for $\tau=200 \mathrm{ps}$ \\
\hline $9.52 \times 10^{-9}\left[\mathrm{~m}^{2} \mathrm{~K} / \mathrm{W}\right]$ & $4.45 \times 10^{-8}\left[\mathrm{~m}^{2} \mathrm{~K} / \mathrm{W}\right]$ & $8.91 \times 10^{-9}\left[\mathrm{~m}^{2} \mathrm{~K} / \mathrm{W}\right]$ \\
\hline
\end{tabular}

For the hypothetical case in which the water thermal expansion is assumed temperature independent, the maximum pressure variations is found to be roughly proportional to the effective absorption coefficient over a wide spectral range $(400-1500 \mathrm{~nm})$, with small deviations (8-20\%) for wavelengths approaching the surface plasmon resonance (SPR).

On the contrary, accounting for the temperature dependence of water thermal expansion, a non-linear relation between the PA signal and the effective absorption coefficient is found for wavelengths tuned at the SPR. In the proximity of the SPR, the non-linearity is found to increase the PA signal by more than 50\%, while non-linear effects are negligible in the NIR. The analysis proves that an adequate choice of the optical wavelength either suppresses or enhances the non-linearity affecting the PA signal.

The PA signal is found to be affected by the NS size only at SPR, where the PA pressure variations increase with increasing NS diameter. This effect, which can be ascribed to a combination of size effects on the optical absorption cross section and on the contact surface between the heated NS and the surrounding water (which thermally expands and launches the largest part of the PA pressure wave), together with effects of changing thermal boundary conduction, might explain observations in literature of PA signal magnitude enhancement [20] (or, according to Pang et al. [43], reduction) after coating Au nanoparticles with a silica layer. We also investigated the effect of the TBC on the PA signal. In the case of a 5 ns laser pulse, the PA signal is not affected by the TBC, the opposite being true for a 200 ps laser pulse.

Although in our computations the water light absorption is limited to a small shell around the NS, yielding excitation of high frequencies, the obtained pressure variations allow to evaluate the PA signal of a real sample. This is done by simply adding up the pressure variations generated by the core-shell light absorbing domain, keeping into account the correct phase. For this reason, the conclusions concerning the proportionality between the PA signal and $\beta_{\text {eff, }}$, the non-linear effects, the NS size and TBC are extendible to the PA signal generated by a real sample.

The present work provides experimentalists a theoretical tool to establish values of the size of the nanoparticles, the optical wavelength and the laser pulse duration that are optimum for efficient PA signal generation. The importance of our findings goes beyond the specific case here presented. For instance, the results could be beneficial for the implementation of new generation multi-spectral PA imaging techniques. Furthermore, the same modelling could be easily adapted to investigate the PA generation in interfacial liquids or polymers layers via nanopatterned metallic arrays $[46,47]$, an emerging technique to access the mechanical properties of interfacial fluid layers.

\section{Conflict of interest}

The authors declare that there are no conflicts of interest.

\section{Declaration of Competing Interest}

The authors report no declarations of interest.

\section{Acknowledgments}

Christ Glorieux and Marco Gandolfi are grateful to the KU Leuven Research Council for financial support (C14/16/063 OPTIPROBE). Francesco Banfi acknowledges financial support from Université de Lyon in the frame of the IDEXLYON Project-Programme Investissements d'Avenir (ANR-16-IDEX-0005) and from Université Claude Bernard Lyon 1 thorugh the BQR Accueil EC 2019 grant. Marco Ganolfi acknowledges financial support from Università Cattolica del Sacro Cuore in the frame of the dual supervision PhD. Francesco Banfi and Marco Gandolfi acknowledge financial support from the MIUR Futuro in ricerca Grant in the frame of the ULTRA - NANO Project (Project No. RBFR13NEA4). Marco Gandolfi acknowledges financial support from the CNR Joint Laboratories program 2019-2021. 


\section{Appendix A. Supplementary data}

Supplementary data associated with this article can be found, in the online version, at https://doi.org/10.1016/j.pacs.2020.100199.

\section{References}

[1] A.G. Bell, Art. xxxiv.-on the production and reproduction of sound by light, Am. J. Sci. 20 (118) (1880) 305 (1880-1910).

[2] F.A. McDonald, Photoacoustic effect and the physics of waves, Am. J. Phys. 48 (1) (1980) 41-47.

[3] S. Volz, J. Ordonez-Miranda, A. Shchepetov, M. Prunnila, J. Ahopelto, T. Pezeril, G. Vaudel, V. Gusev, P. Ruello, E.M. Weig, et al., Nanophononics: state of the art and perspectives, Eur. Phys. J. B 89 (1) (2016) 15.

[4] O. Matsuda, M.C. Larciprete, R. Li Voti, O.B. Wright, Fundamentals of picosecond laser ultrasonics, Ultrasonics 56 (2015) 3-20.

[5] M. Xu, L.V. Wang, Photoacoustic imaging in biomedicine, Rev. Sci. Instrum. 77 (4) (2006) 041101.

[6] E. Zhang, J. Laufer, R. Pedley, P. Beard, In vivo high-resolution 3d photoacoustic imaging of superficial vascular anatomy, Phys. Med. Biol. 54 (4) (2009) 1035.

[7] D. Wu, L. Huang, M.S. Jiang, H. Jiang, Contrast agents for photoacoustic and thermoacoustic imaging: a review, Int. J. Mol. Sci. 15 (12) (2014) 23616-23639.

[8] S. Egerev, Y.O. Simanovskii, A. Pashin, Radiaton of axisymmetric cavitation sound source induced by a laser pulse, Advances in Nonlinear Acoustics, World Scientific, Singapore, 1993, pp. 436-442.

[9] S. Alimpiev, Y.O. Simanovskii, S. Egerev, A. Pashin, Optoacoustic detection of microparticles in liquids at laser fluences below the optical breakdown threshold, Laser Chem. 16 (2) (1995) 63-73.

[10] A.A. Karabutov, E.V. Savateeva, A.A. Oraevsky, Optoacoustic supercontrast for early cancer detection, Biomedical Optoacoustics II vol. 4256, International Society for Optics and Photonics, 2001, pp. 179-187.

[11] S. Egerev, O. Ovchinnikov, A. Fokin, Optoacoustic conversion in suspensions: the competition of mechanisms and statistical characteristics, Acoust. Phys. 51 (2) (2005) 160-166.

[12] X. Yang, S.E. Skrabalak, Z.-Y. Li, Y. Xia, L.V. Wang, Photoacoustic tomography of a rat cerebral cortex in vivo with au nanocages as an optical contrast agent, Nano Lett. 7 (12) (2007) 3798-3802.

[13] M. Eghtedari, A. Oraevsky, J.A. Copland, N.A. Kotov, A. Conjusteau, M. Motamedi, High sensitivity of in vivo detection of gold nanorods using a laser optoacoustic imaging system, Nano Lett. 7 (7) (2007) 1914-1918.

[14] A.A. Oraevsky, A.A. Karabutov, E.V. Savateeva, Enhancement of optoacoustic tissue contrast with absorbing nanoparticles, Hybrid and Novel Imaging and New Optical Instrumentation for Biomedical Applications vol. 4434, International Society for Optics and Photonics, 2001, pp. 60-69.

[15] S. Mallidi, T. Larson, J. Tam, P.P. Joshi, A. Karpiouk, K. Sokolov, S. Emelianov, Multiwavelength photoacoustic imaging and plasmon resonance coupling of gold nanoparticles for selective detection of cancer, Nano Lett. 9 (8) (2009) 2825-2831.

[16] K.L. Kelly, E. Coronado, L.L. Zhao, G.C. Schatz, The Optical Properties of Metal Nanoparticles: The Influence of Size, Shape, and Dielectric Environment, (2003).

[17] A. Crut, P. Maioli, N. Del Fatti, F. Vallée, Optical absorption and scattering spectroscopies of single nano-objects, Chem. Soc. Rev. 43 (11) (2014) 3921-3956.

[18] W. Li, X. Chen, Gold nanoparticles for photoacoustic imaging, Nanomedicine 10 (2) (2015) 299-320.

[19] Y.-S. Chen, W. Frey, S. Aglyamov, S. Emelianov, Environment-dependent generation of photoacoustic waves from plasmonic nanoparticles, Small 8 (1) (2012) 47-52.

[20] Y.-S. Chen, W. Frey, S. Kim, P. Kruizinga, K. Homan, S. Emelianov, Silica-coated gold nanorods as photoacoustic signal nanoamplifiers, Nano Lett. 11 (2) (2011) 348-354.

[21] D. Kumar, D.P. Ghai, R. Soni, Simulation studies of photoacoustic response from gold-silica core-shell nanoparticles, Plasmonics 13 (6) (2018) 1833-1841.

[22] Y. Shi, S. Yang, D. Xing, Quantifying the plasmonic nanoparticle size effect on photoacoustic conversion efficiency, J. Phys. Chem. C 121 (10) (2017) 5805-5811.

[23] H.-Q. Yu, J. Yao, X.-W. Wu, D.-J. Wu, X.-J. Liu, Tunable photoacoustic properties of gold nanoshells with near-infrared optical responses, J. Appl. Phys. 122 (13) (2017) 134901.

[24] H. Ju, R.A. Roy, T.W. Murray, Gold nanoparticle targeted photoacoustic cavitation for potential deep tissue imaging and therapy, Biomed. Optics Express 4 (1) (2013) 66-76.

[25] C.-W. Wei, M. Lombardo, K. Larson-Smith, I. Pelivanov, C. Perez, J. Xia, T. Matula, D. Pozzo, M. O'Donnell, Nonlinear contrast enhancement in photoacoustic molecular imaging with gold nanosphere encapsulated nanoemulsions, Appl. Phys. Lett. 104 (3) (2014) 033701.

[26] P. Beard, Biomedical photoacoustic imaging, Interface Focus 1 (4) (2011) 602-631.
[27] V. Gujrati, A. Mishra, V. Ntziachristos, Molecular imaging probes for multi-spectral optoacoustic tomography, Chem. Commun. 53 (34) (2017) 4653-4672.

[28] M.B. Fonseca, L. An, B.T. Cox, Sulfates as chromophores for multiwavelength photoacoustic imaging phantoms, J. Biomed. Optics 22 (12) (2017) 125007.

[29] V. Ntziachristos, D. Razansky, Molecular imaging by means of multispectral optoacoustic tomography (MSOT), Chem. Rev. 110 (5) (2010) 2783-2794.

[30] L. Meng, O. Deschaume, L. Larbanoix, E. Fron, C. Bartic, S. Laurent, M. Van der Auweraer, C. Glorieux, Photoacoustic temperature imaging based on multi-wavelength excitation, Photoacoustics 13 (2019) 33-45.

[31] G.A. Pang, J. Laufer, R. Niessner, C. Haisch, Photoacoustic signal generation in gold nanospheres in aqueous solution: signal generation enhancement and particle diameter effects, J. Phys. Chem. C 120 (48) (2016) 27646-27656.

[32] M. Ferrera, G. Della Valle, M. Sygletou, M. Magnozzi, D. Catone, P. O’Keeffe, A. Paladini, F. Toschi, L. Mattera, M. Canepa, et al., Thermometric calibration of the ultrafast relaxation dynamics in plasmonic Au nanoparticles, ACS Photonics (2020).

[33] K. Metwally, S. Mensah, G. Baffou, Fluence threshold for photothermal bubble generation using plasmonic nanoparticles, J. Phys. Chem. C 119 (51) (2015) 28586-28596.

[34] I.G. Calasso, W. Craig, G.J. Diebold, Photoacoustic point source, Phys. Rev. Lett. 86 (16) (2001) 3550.

[35] A. Prost, F. Poisson, E. Bossy, Photoacoustic generation by a gold nanosphere: from linear to nonlinear thermoelastics in the long-pulse illumination regime, Phys. Rev. B 92 (11) (2015) 115450.

[36] G.A. Pang, L. Qiu, C. Haisch, J. Laufer, Towards biochemical sensing with gold nanoparticles through suppression of nonlinear photoacoustic signal generation, Photons Plus Ultrasound: Imaging and Sensing 2019 vol. 10878, International Society for Optics and Photonics, 2019, p. 108786 Q.

[37] M. Gandolfi, A. Crut, F. Medeghini, T. Stoll, P. Maioli, F. Vallée, F. Banfi, N. Del Fatti, Ultrafast thermo-optical dynamics of plasmonic nanoparticles, J. Phys. Chem. C 122 (15) (2018) 8655-8666.

[38] T. Stoll, P. Maioli, A. Crut, S. Rodal-Cedeira, I. Pastoriza-Santos, F. Vallée, N. Del Fatti, Time-resolved investigations of the cooling dynamics of metal nanoparticles: impact of environment, J. Phys. Chem. C 119 (22) (2015) 12757-12764.

[39] F. Banfi, V. Juvé, D. Nardi, S. Dal Conte, C. Giannetti, G. Ferrini, N. Del Fatti, F. Vallée, Temperature dependence of the thermal boundary resistivity of glassembedded metal nanoparticles, Appl. Phys. Lett. 100 (1) (2012) 011902.

[40] C. Caddeo, C. Melis, A. Ronchi, C. Giannetti, G. Ferrini, R. Rurali, L. Colombo, F. Banfi, Thermal boundary resistance from transient nanocalorimetry: a multiscale modeling approach, Phys. Rev. B 95 (8) (2017) 085306.

[41] R. Stoner, H. Maris, Kapitza conductance and heat flow between solids at temperatures from 50 to 300 k, Phys. Rev. B 48 (22) (1993) 16373.

[42] Y. Shi, H. Qin, S. Yang, D. Xing, Thermally confined shell coating amplifies the photoacoustic conversion efficiency of nanoprobes, Nano Res. 9 (12) (2016) 3644-3655.

[43] G.A. Pang, F. Poisson, J. Laufer, C. Haisch, E. Bossy, Theoretical and Experimental Study of Photoacoustic Excitation of Silica-Coated Gold Nanospheres in Water, (2019) (arXiv preprint), arXiv:1905.06227.

[44] P. Maioli, T. Stoll, H.E. Sauceda, I. Valencia, A. Demessence, F. Bertorelle, A. Crut, F. Vallee, I.L. Garzón, G. Cerullo, et al., Mechanical vibrations of atomically defined metal clusters: from nano-to molecular-size oscillators, Nano Lett. 18 (11) (2018) 6842-6849.

[45] G.M. Hale, M.R. Querry, Optical constants of water in the $200-\mathrm{nm}$ to $200-\mu \mathrm{m}$ wavelength region, Appl. Optics 12 (3) (1973) 555-563.

[46] M. Travagliati, D. Nardi, C. Giannetti, V. Gusev, P. Pingue, V. Piazza, G. Ferrini, F. Banfi, Interface nano-confined acoustic waves in polymeric surface phononic crystals, Appl. Phys. Lett. 106 (2) (2015) 021906.

[47] D. Nardi, M. Travagliati, M.M. Murnane, H.C. Kapteyn, G. Ferrini, C. Giannetti, F. Banfi, Impulsively excited surface phononic crystals: a route toward novel sensing schemes, IEEE Sens. J. 15 (9) (2015) 5142-5150.

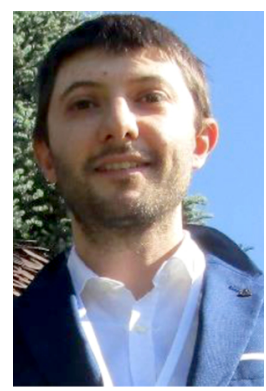

Marco Gandolfi obtained his MSc degree in Physics at Università Cattolica del Sacro Cuore (Italy) in 2014. Then he participated in a joint $\mathrm{PhD}$ project between KU Leuven (Belgium) and Università Cattolica del Sacro Cuore. He got his $\mathrm{PhD}$ in Physics in 2019. He currently works as a postdoctoral researcher at the National Institute of Optics (CNRINO), in Italy. His interests cover the modelling of optics, thermics, mechanics and acoustics at the nanoscale. 


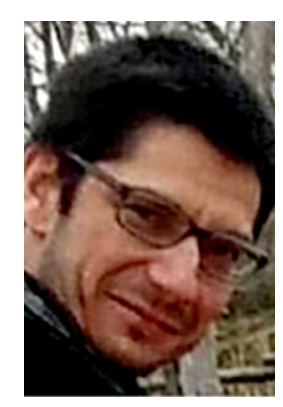

Francesco Banfi earned his Ph.D. degree in physics from Università degli Studi of Pavia, Italy, in 2004. He is professor of physics at Université Claude Bernard Lyon 1 where

he works at the Institute Lumière Matière. His scientific interests are in the field of ultrafast energy transients at the nanoscale. In the past years, his main contributions have been in the field of nano-scale mechanics and nano-scale heat transfer investigated by ultrafast optical techniques.

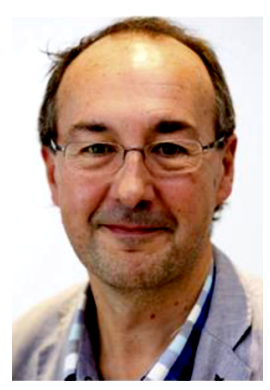

Christ Glorieux obtained his $\mathrm{PhD}$ degree in 1994 on the topic "Depth profiling of inhomogeneous materials and study of the critical behaviour of gadolinium by photoacoustic and related techniques," from KU Leuven. Now he is an Associate Professor at the Laboratory of Soft Matter and Biophysics, KU Leuven. His research interests include photothermal applications, adiabatic scanning calorimetry and laser ultrasonics for the fundamental study of the thermophysical properties of complex soft and biological matter, the development of measurement techniques for characterization and depth profiling of thin (sub-micron) layered structures, non-destructive evaluation, physical acoustics, room acoustics, building acoustics and environmental acoustics. In March 2020, 257 articles (co-)authored

by him were listed on Web of Science. 